\title{
Optimization of viral protein ratios for production of rAAV serotype 5 in the baculovirus system
}

\author{
Bas Bosma ${ }^{1} \cdot$ Francois du Plessis $^{1} \cdot$ Erich Ehlert $^{1} \cdot$ Bart Nijmeijer $^{1} \cdot$ Martin de Haan $^{1} \cdot$ Harald Petry $^{1} \cdot$ Jacek Lubelski $^{1}$
}

Received: 28 January 2018 / Revised: 21 May 2018 / Accepted: 14 June 2018 / Published online: 1 August 2018

(c) The Author(s) 2018. This article is published with open access

\begin{abstract}
Recombinant adeno-associated virus (rAAV) has become the vector of choice for the development of novel human gene therapies. High-yield manufacturing of high-quality vectors can be achieved using the baculovirus expression vector system. However, efficient production of rAAV in this insect cell-based system requires a genetic redesign of the viral protein 1 (VP1) operon. In this study, we generated a library of rationally designed rAAV serotype 5 variants with modulations in the translation-initiation region of VP1 and investigated the potency of the resulting vectors. We found that the initiation strength at the VP1 translational start had downstream effects on the VP2/VP3 ratio. Excessive incorporation of VP3 into a vector type decreased potency, even when the VP1/VP2 ratio was in balance. Finally, we successfully generated a potent rAAV vector based on serotype 5 with a balanced VP1/VP2/VP3 stoichiometry.
\end{abstract}

\section{Introduction}

Adeno-associated virus (AAV) has been used as a gene delivery vehicle in numerous clinical trials and is an integral component of the first approved gene therapy medicine in Europe, alipogene tiparvovec (Glybera ${ }^{\circledR}$ uniQure). The wildtype (WT) AAV genome comprises inverted terminal repeats that flank two open reading frames (ORFs): rep and cap. The rep ORF includes four overlapping genes encoding the proteins required for AAV replication and packaging, whereas the cap ORF contains overlapping sequences encoding viral proteins 1, 2, and 3 (VP1-VP3), which interact to form a capsid. Recombinant AAV (rAAV) is constructed by replacing the rep and cap genes with a therapeutic gene; consequently, an adequate source of rep and cap proteins must be provided in trans for the rAAV lifecycle to proceed.

The baculovirus expression vector system (BEVS), which has been widely and successfully used for the expression of a variety of heterologous proteins, has proved effective for generating clinical-quality rAAV in quantities necessary for industrial-scale production [1]. The ground-breaking use of

Jacek Lubelski

J.Lubelski@uniqure.com

1 Vector Development department, research at uniQure, Paasheuvelweg 25A, 1105 BP Amsterdam, The Netherlands baculoviruses as helper and delivery vectors for rAAV in insect cells was first reported by Urabe et al. [2]. Initially, rAAV was produced by coinfecting insect cells with three separate baculoviruses: Bac-Rep, Bac-Cap, and Bac-Vec. In order to generate infectious rAAV vectors in insect cells, both the Cap and Rep proteins must be expressed at appropriate levels. To achieve this, two main modifications were performed.

Under normal conditions, large Rep78 and small Rep52 derive from two distinct promoters ( $\mathrm{p} 5$ and $\mathrm{p} 19$, respectively). The transcribed mRNAs then undergo splicing to form Rep68 and Rep40. To ensure strong expression of both Rep78 and Rep52, Urabe and colleagues constructed a DNA cassette in which the expression of all four Rep proteins was driven by separate baculovirus promoters.

The next challenge relates to the expression of the capsid proteins. WT AAV expresses VP1-3 from its $\mathrm{p} 40$ promoter and the transcribed mRNA is spliced into two species: [3, 4] one is responsible for VP1 expression, and the other for both VP2 and VP3 expression. The production of VP2 and VP3 from the same mRNA occurs via a "leaky ribosomal scanning mechanism", in which the protein is initiated from a noncanonical start codon, i.e., ACG. This start codon is occasionally missed by the ribosome complex. If this occurs, the ribosome proceeds until it finds the canonical start of VP3. However, because the splicing machinery differs between vertebrate and insect cells, this mechanism does not result in the generation of infectious capsids in insect cells. To 
overcome this challenge, Urabe et al. [2]. modified the translational start (Kozak sequence) of VP1 of AAV serotype 2 to resemble that of the noncanonical VP2 start codon. The translational start of VP1 was changed to ACG and the initiation sequence, which consists of nine nucleotides upstream of the VP1 start codon, was changed to the proceeding VP2 start codon. This modification resulted in the expression of appropriate levels of the three VPs and led to the assembly of infectious serotype 2 capsids from a single polycistronic mRNA [2].

In order to successfully and efficiently produce novel rAAV serotypes in BEVS, similar adaptations to the cap operon adaptations are required [5]. However, the various serotypes differ in their genetic context surrounding the VP initiation sequences, which may influence VP expression levels and particle infectivity. It has previously been demonstrated that expression of serotype 1 in insect cells requires fine tuning to result in high-potency vectors [6]. Earlier attempts to adapt AAV serotype 5 for production using BEVS were only partially successful, due to the low levels of VP1 incorporation into the capsid [7, 8]. To circumvent this problem, Urabe et al. [4]. generated a chimeric type $2 / 5$ virus comprising the $\mathrm{N}$-terminal 136 amino acids from serotype 2 combined with the remainder sequence from serotype 5 . This chimeric virus was efficiently produced and displayed a similar potency to WT AAV5 produced in mammalian HEK293T cells [7]. Although successful, this virus does not represent the rAAV5 serotype.

To better understand the effects of rAAV VP expression ratios on infectivity, we generated a library of novel cap 5 designs using leaky ribosomal scanning mechanisms in a BEVS-based manufacturing platform. As previously reported [9-14], we found that low levels of VP1 incorporation into AAV particles negatively influenced the potency of the vector, and that (more generally) the stoichiometry of all three VPs was crucial for the vector infectivity. The potency of our novel vector variants was negatively correlated with the level of VP3 incorporation; high levels of VP3 were associated with poor transduction, both in vitro and in vivo. Finally, we identified in a nonchimeric rAAV5 capsid with an VP1/VP2/ VP3 stoichiometry similar to that found in the WT virus, which displayed a higher potency than that previously reported for chimeric rAAV5 in insect cells [7, 8].

\section{Results}

\section{Modification of the translation-initiation start site and downstream context sequence (Constructs 1-6) alters the stoichiometry of VP1:VP2:VP3}

The introduction of the noncanonical start codon (ACG) in Construct 1, and the 9-nucleotide (CCTGTTAAG) upstream context of VP2 previously reported by Urabe et al. resulted in low levels of VP1 incorporation compared to WT (Fig. 1a, b). A similar stoichiometry was observed for Construct 3, which included a guanine residue at position +4 , designed to increase the homology of this region with the canonical Kozak sequence. Low levels of VP1 incorporation were also noted for Constructs 4-6. In each of these constructs, another noncanonical codon (CTG) was introduced in combination with the upstream CCTGTTAAG sequence. (In Construct 4 , these modifications were combined with the mutation of a second serine residue to threonine; in Construct 6, TC was replaced with AG at positions +4 and +5 without altering the primary amino acid sequence and, in Construct 5, ACT was inserted as a second triplet with the modification of the original +2 triplet to AGC). An alanine residue was inserted at position +2 of Construct 2 to increase the homology of this region with the canonical Kozak sequence. The combination of the additional alanine-forming guanine at nucleotide position +4 , the noncanonical ACG, and the upstream insertion of CCTGTTAAG resulted in a 1:1 stoichiometry of VP1/VP2, with high levels of VP3 incorporation (Fig. 1a, b).

\section{Reducing the length of the promotor sequence (Constructs 7-12) decreases the VP1:VP2 ratio}

Constructs 7-12 were designed to match Constructs 1-6, with subtle differences (Table 1). For example, the only difference between Constructs 7 and 2 was the shorter promotor sequence in Construct 7. This change resulted in a decrease in VP1:VP2 ratio (Fig. 1). A similar decrease was observed with Construct 8 , which had the same sequence as Construct 7 minus the initiation region upstream of VP2.

To preserve the positive influence of the combination of noncanonical ACG with the additional alanine whilst increasing the expression/incorporation of VP1/2 in relation to VP3, additional combinations of mutants were tested. To increase translation-initiation, the translationinitiation start site for VP1 in Construct 2 was mutated to the WT ATG sequence (Construct 9). Several weaker initiation triplets TTG (Construct 10), CTG (Construct 11), GTG (Construct 12) were also introduced. Other than Construct 11, rAAV production was not observed for any of these constructs. Interestingly, a combination of CTG as a noncanonical VP1 initiation start and the addition of a GCT triplet (encoding an extra alanine) immediately after the translational start in Construct 11 resulted in higher incorporation of VP1 than VP2 in combination with the attenuation of VP3. This ultimately resulted in a balanced VP stoichiometry similar to that observed with WT AAV5 (Fig. 1a, b). 

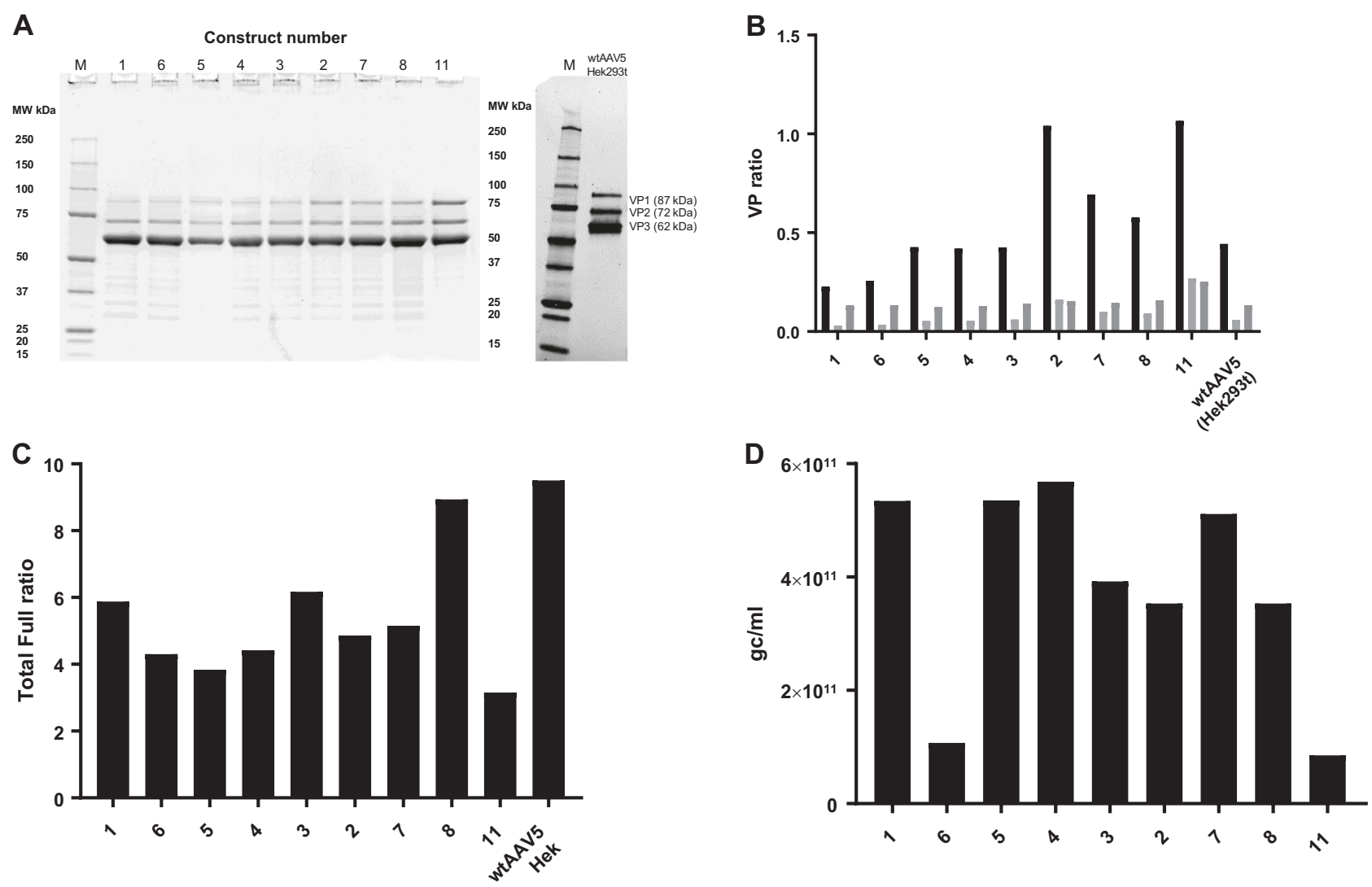

Fig. 1 a Mutant and wild-type AAV5 capsid proteins (VP1-VP3) were purified and resolved on a NuPage gel. b Densitometric analysis of VP1-VP3 bands was conducted via peak integration using a rolling ball algorithm in ImageQuant TL software (GE healthcare). Densitometric analysis was based on one AAV batch per construct. All the

\section{Increased levels of VP3 incorporation are associated with decreased potency of rAAV serotype 5 produced in BEVS}

The potency of the capsids generated using Constructs $1-8$, and 11 was initially studied in vitro using two different continuous liver cell lines: HepG2 (Fig. 2a) and Huh7 (Fig. 2b). In both cell lines, variants with a low VP1:VP2 ratio and an excessive incorporation of VP3 (Constructs 1 and 3-6) displayed low levels of potency. The potency of the vector was increased by balancing the VP1:VP2 ratio (Construct 2). Removing the cloning remnants from the promoter region (Construct 7) and deleting the initiator context (Construct 8) had a negative effect on vector potency. The most potent construct was Construct 11, which had a 1:1 VP1:VP2 ratio and significantly lower levels of VP3 incorporation relative to the earlier constructs. Comparing the potency of construct $11 \mathrm{rAAV}$ produced in BEVS with that of WT AAV5 produced in HEK293T in Huh7 cells at three different multiplicity of infections (MOI) clearly demonstrates comparability (Fig. 2c). batches used in the experiments were produced in the same production round. c Total: full ratio of produced mutant and wild-type AAV batches. d Production yields in $\mathrm{gc} / \mathrm{ml}$ of produced mutant and wildtype AAV batches. MW molecular weight; $\mathrm{kDa}$ kilodaltons; $\mathrm{M}$ marker; VP viral protein

\section{Modifying the VP1 levels have no significant effect on neither the total to full ratio nor the production quantity}

It has been noted previously in AAV8 preparations that the increase of VP1 in the capsid stoichiometry resulted in reduced packaging efficiency [15]. We have, therefore, analyzed the total to full ratio for all constructs generated here (Fig. 1c) as well as determined the overall yield obtained for each construct (Fig. 1d). We observed no correlation between the modification of the VP1 content of each capsid and the total:full ratio. Furthermore, we also do not observe any correlation between the total production yield and the VP1 content of the various constructs.

\section{Infectivity was reduced by low incorporation of VP1 and excessive incorporation of VP3}

Infectivity refers to the number of DNA containing virus particles required to constitute one infectious event. However, the assay described here is rather defined by the 
Table 1 Description of rAAV5 capsid variants

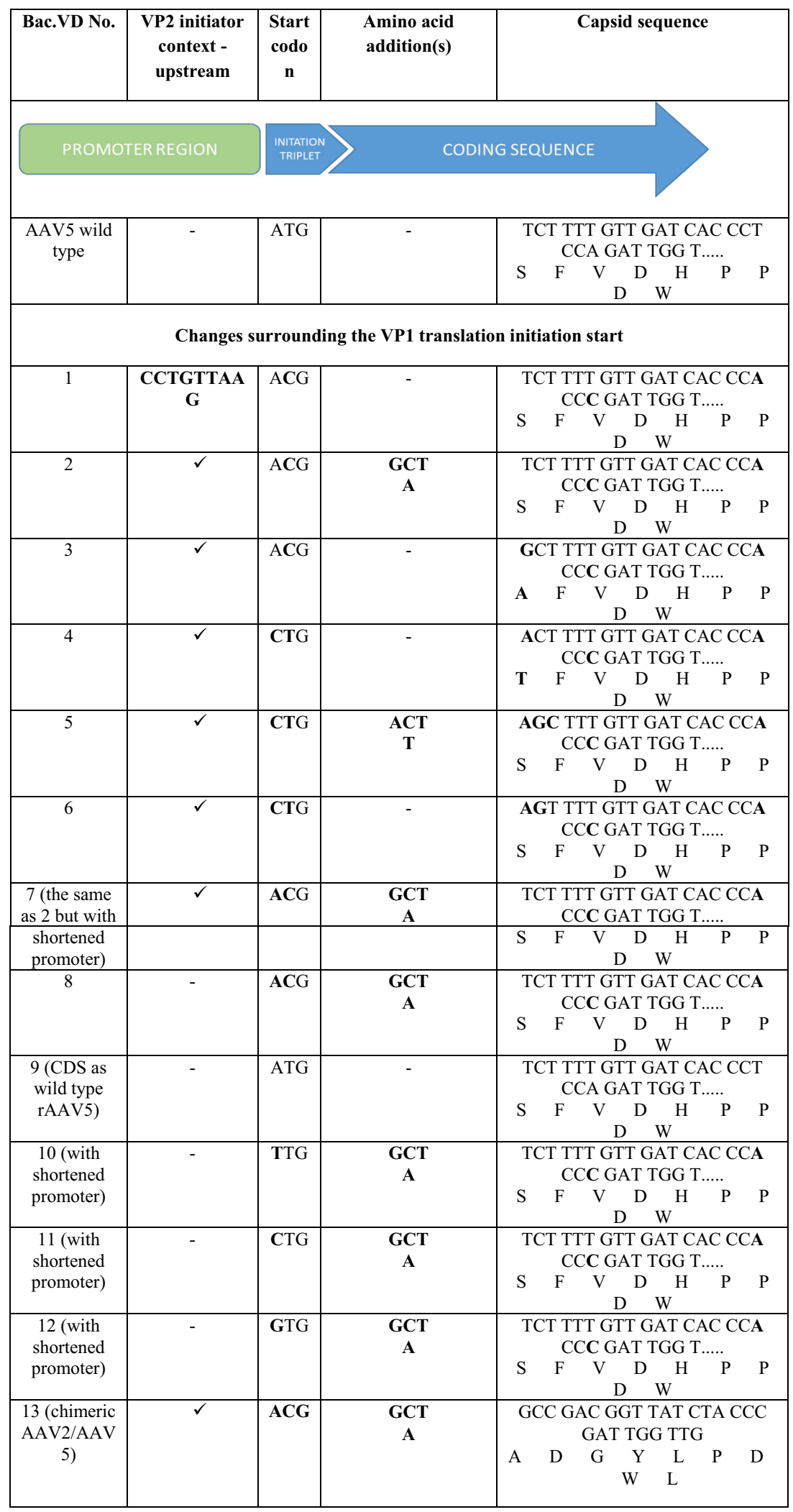

Mutations surrounding the translational start of VP1 were generated in order to vary the stoichiometry of the three VPs expressed in insect cells Nucleotides/amino acids that were altered compared to the WT serotype 5 capsid sequence are indicated in bold

$V P$ viral protein; $C D S$ coding sequence; $r A A V$ recombinant adeno-associated virus; Bac baculovirus 
Fig. 2 In vitro potency of capsid mutants carrying the seap expression cassette in a Hela cells $\left(\right.$ MOI $\left.5 \times 10^{3}\right)$ and b Huh7 cells $\left(\right.$ MOI $\left.5 \times 10^{3}\right)$. c Hek293t wtAAV5 and BEVs produced AAV5 (MOI $5 \times 10^{3}$, construct 11) in Huh7 cells. The activity of the reporter gene is measured indirectly as emission of light and is expressed in relative light units (RLU). Error bars in the graphs represent the standard deviations and are based on three replicate seap activity measurements in the same experiment. Construct 2 and 11 were compared via a $t$ test. a.u., arbitrary units, relative light units (RLU)
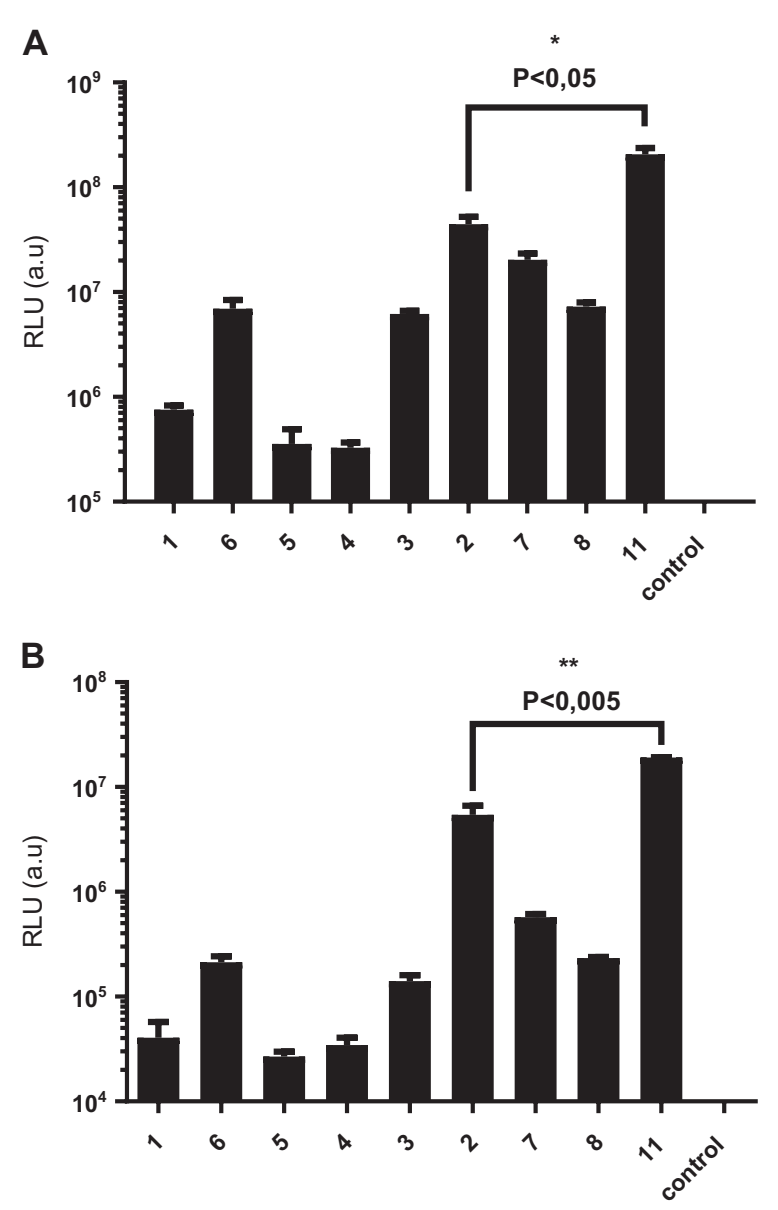

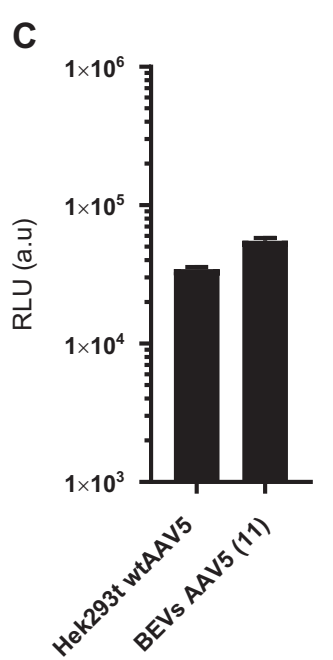

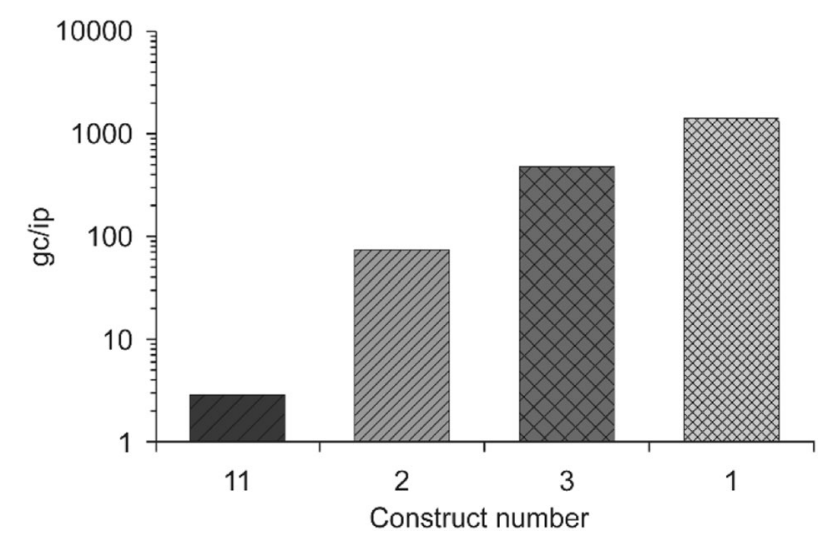

Fig. 3 Infectivity in gc/ip of selected rAAV5 capsid variants as measured in an in vitro limiting dilution assay in HelaRC32 cells. HelaRC32 cells were infected at starter dilutions of $200 \mathrm{gc} / \mathrm{well}$ (Construct 11), $1000 \mathrm{gc} /$ well (Construct 2 and 3) and 10,000 gc/well (construct 1), respectively. A low gc/ip ratio indicates high infectivity. See Methods for assay description. gc genome copies; ip infectious particles

detection of the event where a DNA containing virus managed to successfully reach the nucleus of the cell and concomitantly gets amplified by the AAV replicase that is integrated into the HelaRC32 DNA. By performing a limited dilution, we can express the genome copies needed on average to successfully constitute an infectious event. We postulated that an increased VP1:VP3 stoichiometry would enable the virus to require less particles to be infective. This is directly linked to the presence of the phospholipase 2 domain on the VP1-unique sequence. The domain has been shown to be responsible for the escape of the AAV virus from the endosome $[16,17]$.

Compared to Constructs 2 (equal ratio of VP1/VP2 but greater VP3), 3 and 1 (low VP1 and high VP3), Construct 11 (WT-like stoichiometry) was associated with higher levels of infectivity (Fig. 3). See the Methods section for a description of the infectivity assay.

\section{Balancing VP stoichiometry improved gene transfer in mice}

Based on the in vitro results, Constructs 2 and 11 were selected for in vivo testing in mice. The chimeric type $2 / 5$ construct (Construct 13) developed by Urabe et al. [4] was 


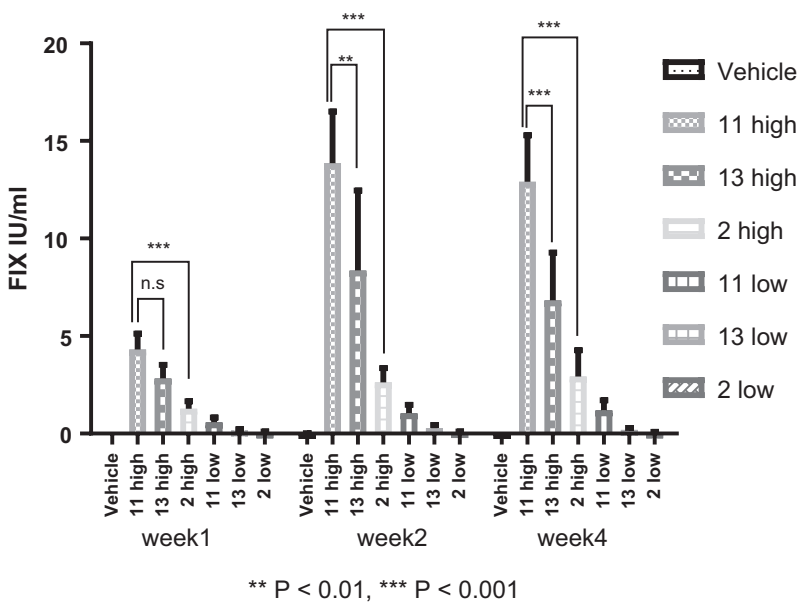

Fig. 4 In vivo potency of selected rAAV5 capsid variants in rodents. Plasma concentrations of hFIX in C57BL/6 mice following i.v. administration. FIX expression was monitored in mice upon administration of three different vectors, i.e., capsid variant rAAV5 (Construct 2), rAAV5 (Construct 11), and rAAV5 (Construct 13), containing the hFIX expression cassette. Mice were injected with two different doses (low dose, $2 \mathrm{e} 12 \mathrm{gc} / \mathrm{kg}$; high dose, $2 \mathrm{e} 13 \mathrm{gc} / \mathrm{kg}$ ). hFIX was measured in plasma 1,2 , and 4 weeks postinjection by means of a specific hFIX ELISA. Each line represents the average of five animals in each dose group. Statistical analysis was performed using one-way ANOVA. IU international unit; hFIX human Factor IX; rAAV recombinant adeno-associated virus

included in this study as a reference control. Construct 11 displayed improved potency demonstrated by higher circulating hFIX concentrations compared to Constructs 2 and 13 (Fig. 4).

\section{Balancing VP stoichiometry improved gene transfer in NHPs}

Based on results in mice, Constructs $2\left(5 \times 10^{12} \mathrm{gc} / \mathrm{kg}\right)$ and $11\left(5 \times 10^{11}\right.$ and $\left.5 \times 10^{12} \mathrm{gc} / \mathrm{kg}\right)$ were tested for in vivo potency in NHPs. All predosing samples returned values below the lower limit of quantification, demonstrating assay specificity. For each construct, the level of hFIX protein peaked around 1-week postdosing and stabilized approximately 7 weeks thereafter (Fig. 5). The higher dose of Construct 11 resulted in a higher expression of hFIX protein than the same dose of Construct 2. Furthermore, the level of hFIX expression from the tenfold lower dose of Construct 11 was only slightly lower that that driven by the higher dose of Construct 2.

Vector DNA was detected in the liver of all tested NHPs (Fig. 6). High-dose Construct 11 resulted in approximately tenfold higher delivery of transgene DNA than the matching dose of Construct 2. Furthermore, the concentration of vector DNA delivered to liver by high-dose Construct 2 was similar to that observed with the tenfold lower dose of Construct 11.

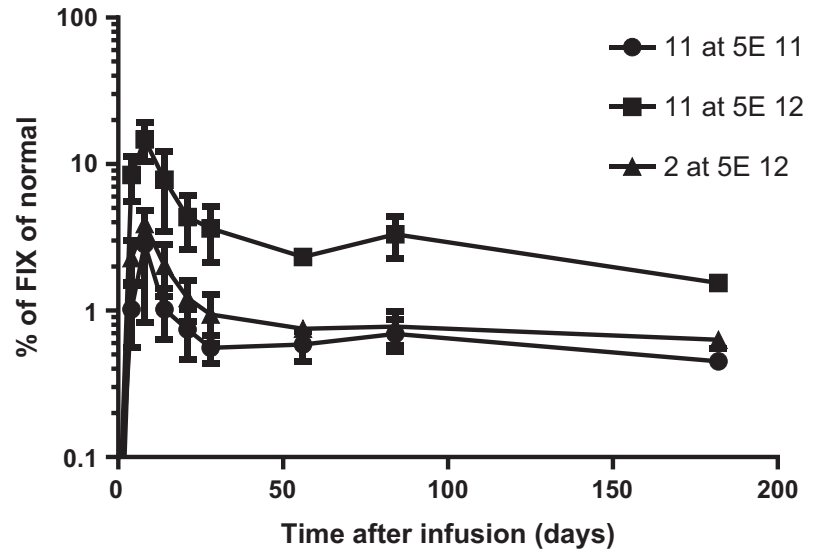

Fig. 5 In vivo potency in nonhuman primates. Plasma concentrations of hFIX in cynomolgus monkeys following i.v. administration of Constructs 11 (5e11 and $5 \mathrm{e} 12 \mathrm{gc} / \mathrm{kg}$ ), and $2(5 \mathrm{e} 12 \mathrm{gc} / \mathrm{kg})$. hFIX levels was measured in plasma 1, 2, and 4 weeks postinjection by means of a specific hFIX ELISA. Each line represents the average of three animals in each dose group. Error bars represent standard error of mean. hFIX human Factor IX

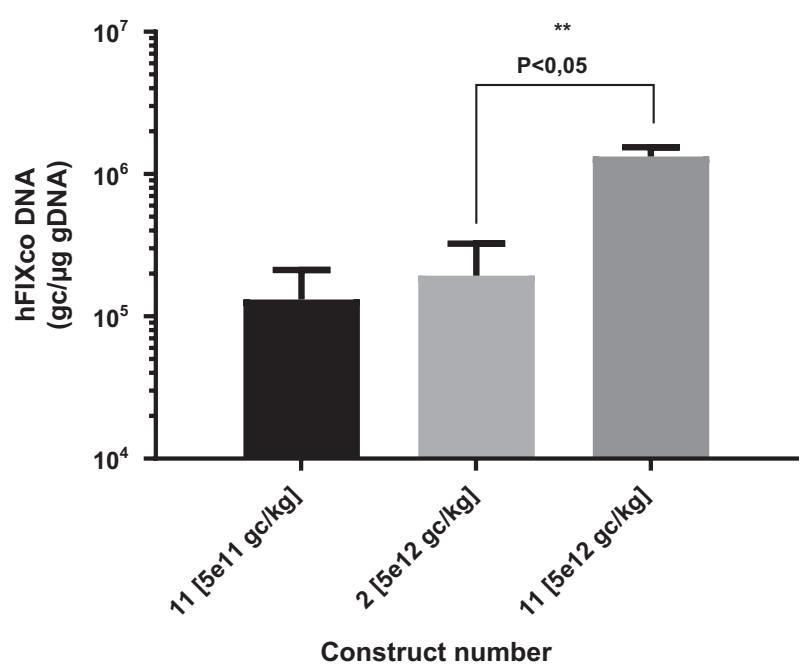

Fig. 6 Average vector DNA levels in nonhuman primate liver tissue measured by specific Q-PCR. Each bar represents an average of three animals. Statistics was performed using a paired $t$ test. hFIX human Factor IX; gc genome copies; gDNA genomic DNA

\section{Discussion}

Production of rAAV in insect cells requires fine tuning of the cap operon. In mammalian cells, AAV expresses the capsid proteins from a single ORF via alternative splicing and a leaky ribosomal scanning mechanism involving the poorly utilized ACG initiator start for VP2. This results in a VP1: VP2:VP3 stoichiometry of 1:1:10. The same ORF used in insect cells fails to produce AAV vectors with a balanced VP stoichiometry $[2,8]$. Urabe et al, overcame this challenge for the rAAV2 serotype by changing the VP1 initiator triplet to 
ACG and by adding the nine nucleotides upstream from the translation-initiation start site. These changes resulted in the production of three capsid proteins with a WT-like stoichiometry. However, the same changes used for an rAAV5 expression cassette resulted in low VP1 and high VP3 capsid subunit ratios that displayed a lower potency than WT.

To investigate the impact of VP1:VP2:VP3 stoichiometry on vector potency in vitro and in vivo, we systematically altered the cap gene to produce rAAV5 variants with different stoichiometries. Construct 1 incorporated several adaptations that had previously been used to successfully generate rAAV2 in insect cells [2]. This mutant contained the 9 nucleotides upstream of the VP2 initiator context placed directly upstream of the VP1 translational start (the noncanonical translationinitiation start codon ACG). This sequence naturally precedes the noncanonical start codon, ACG, of VP2, and we hypothesized that it would provide an optimal upstream context for translation initiation for the noncanonical start of VP1. Unexpectedly, these adaptations resulted in low-potency rAAV5 and a capsid with low levels of VP1 incorporation.

To address this issue, we changed the WT ATG to ACG and CTG, and various mutations were introduced to provide optimal downstream context from the start codon. Most of the mutants showed low incorporation of VP1 and a high VP3 content, resulting in low VP1:VP2 and high VP3:VP1 ratios. We, therefore, sought to find a combination of mutagenic changes that would allow for a strong initiation at VP1 and yet still allow leaky ribosomal scanning. Excessive initiation would potentially result in production of the VP1 protein only, whereas a weak translational start is likely to result in low-VP1 production and excessive VP3 generation. A 1:1 ratio of VP1/VP2 was achieved by Construct 2; however, VP3 incorporation into the vector particles remained relatively high. Of note is that both construct 6 and construct 11 utilize CTG as a start codon. Also, both constructs have an overall yield that is slightly lower when compared to the others (Fig. 1d). As constructs 6 and 11 have vastly different VP1 content (Fig. 1a), we can postulate that the leaky ribosomal scanning mechanism generating these capsids through the CTG start codon effectively lowers or delays VP1 production and concomitantly affects the overall yield. Interestingly, when we are producing rAAV5 with varying VP1 stoichiometries in the BEVS system we are not observing any negative effects on the production efficiency as was observed for rAAV8 [15]. This might be due to either the production of rAAV in BEVS or this is unique to rAAV5 production in general. In fact, of all constructs listed here, construct 11 also displays the lowest total to full ratio which confirms earlier observations by Kondratov et al. and Mietzsch et al. [18, 19]. In comparison, they utilized another variation on the leaky ribosomal scanning mechanism by modifying a Kozak sequence that was introduced upstream of the canonical
ATG start codon. By modifying the $\mathrm{G}+\mathrm{C}$ content of the Kozak sequence, the initiation of translation at the canonical AUG could be successfully manipulated to obtain capsids with WT like stoichiometry. Furthermore, recent developments in producer cell lines [18, 19] and the MonoBac [20] AAV producer systems highlight the strong interest and drive in fully adapting the BEVS and insect cell manufacturing system as a platform for rAAV production.

Finally, Construct 11 resulted in a higher incorporation of VP1 (high VP1:VP2 ratio) and lower incorporation of VP3 than other tested variants (balanced VP3:VP2 ratio), ultimately resulting in a stoichiometry similar to that observed for the WT AAV5 capsid.

The low ratio of VP1:VP2 proteins has previously been postulated to be responsible for low vector potency [9-14, 21]. The N-terminus of VP1, which is normally buried inside the capsid and becomes exposed during intracellular trafficking of the vector, plays an important role in transporting the vector to the nucleus. The phospholipase domain of this region specifically hydrolizes the 2-acyl ester (sn-2) bond of phospholipids that form the endosome lipid bilayer, resulting in the endosomal escape of AAV [21]. Consequently, high levels of VP1 incorporation into the capsid are expected to improve the efficiency of AAV-based DNA transport to the cell nucleus.

Our results show that the changes in VP stoichiometry correlated well with the potency of the vector in vitro and in various in vivo models. It has previously been reported that a low VP1:VP2 ratio has a negative impact on the potency of the virus [2, 7, 14, 22]. Consistent with this observation, Constructs 1 and 3-6, which have a low VP1:VP2 ratio, had the lowest potency. Increasing the VP1:VP2 ratio had a positive effect on potency (Construct 2); however, the greatest potency was obtained from Construct 11, which included further improvements in the VP1:VP2 ratio and decreased levels of VP3 incorporation, resulting in a decreased VP3:VP1 ratio.

Compared to Construct 2, Construct 11 showed an approximate tenfold increase in DNA delivery to the liver of NHPs, and a threefold higher plasma concentration (persistent levels) of FIX protein. Furthermore, a low-dose $(5 \mathrm{e} 11 \mathrm{gc} / \mathrm{kg})$ of Construct 11 resulted in comparable DNA delivery and approximately twofold lower hFIXco expression than that obtained from a tenfold higher dose of Construct 2. This suggests that the molecular make-up of the vector particle is important for its ability to deliver its DNA cargo to the target cells, which ultimately correlates with vector potency.

Increasing the level of VP1 incorporation whilst reducing VP3 expression consistently resulted in the most potent vectors. Because the genetic design of the VP2 and VP3 translational start for production in BEVS is the same as that found in the WT AAV virus, it was not presupposed that the VP2:VP3 ratio had a major impact on 
vector potency. Consequently, alterations in the VP1 translational start were not expected to affect VP2:VP3 ratios. However, we consistently found that alterations of the VP1 translational start context sequences influenced the expression of VP2 and VP3, leading to unwanted high levels of VP3 incorporation. Consistent with this observation, Construct 11, which included modifications designed to reduce VP3 incorporation, was more potent that the other tested constructs.

Interestingly, when comparing construct 2 and 11 in terms of production titers, a very slight decrease in the overall production titers were observed of construct 11 (Fig. 1d). This could possibly be attributed to the weaker CTG start codon that is used in construct 11. Further substantiating this is the comparative yields obtained by construct 6 , which also utilizes the CTG start codon. We found no correlation between the VP1 content and the overall yield of each construct.

We hypothesize that the strong influence on expression of downstream VP2 and VP3 by mutagenic changes of VP1 is related to the translational process itself. Translation is unidirectional in eukaryotes and starts at the 5'end. Ribosomes, once engaged with mRNA, proceed until they find a translational ATG start in an appropriate context to initiate protein synthesis. Weak initiation starts (e.g., ACG or CTG), if surrounded by appropriate nucleotide context, may initiate protein synthesis in a noncanonical manner. This mechanism is called leaky ribosomal scanning and is recently attracting more attention in engineering and manipulation of protein expression [23, 24]. The strength of the leaky ribosomal scanning at VP1 will determine the extent of the ribosomal "leakage" to VP2 and VP3 and the subsequent strength of protein expression. In turn, the differentiated expression of all three capsid components will determine their presence in the final assembled capsid. We postulate that a selection of noncanonical start codons can be utilized in order to drive all native and rAAV serotypes to express in the baculovirus expression system. Each noncanonical start codon will have a greater or lesser effect on the initiation, and therefore translation of VP1, resulting in capsids with varying capsid protein stoichiometries. Likely, various permutations of start codons and initiation contexts need to be tested with various native and recombinant serotypes in order to build a complete picture of the optimal requirements to produce AAV in insect cells with WT stoichiometry.

In conclusion, we have demonstrated the ability to balance and control the strength of translational initiation at VP1 to generate capsids with varying properties in the BEVS system. Fine tuning of the initiation strength at VP1 will lead to the development of a selection of high- potency rAAV variants that display WT-like VP stoichiometry.

\section{Methods}

\section{Generation of the rAAV5 library}

The translational start of VP1 and its immediate surroundings were modified by site-directed mutagenesis. Constructs included various combinations of changes to: (1) The upstream initiation context, (2) the noncanonical start codon, and (3) the downstream initiation context (Table 1).

\section{Vector production}

rAAV5 variants were generated by infecting $\mathrm{SF}+$ insect cells (Protein Sciences Corporation, Meriden, Connecticut, USA) with freshly amplified baculovirus stocks comprising Cap (serotype 5 variant), Rep (replicase) and one of two reporter transgenes (Secreted embryonic alkaline phosphatase [Seap] or human Factor IX [hFIX]). After a $72 \mathrm{~h}$ incubation at $28^{\circ} \mathrm{C}$, cells were lysed with $1 \%$ Triton X-100 for $1 \mathrm{~h}$. Genomic DNA was digested via benzonase (Merck, Darmstadt, Germany) treatment for $1 \mathrm{~h}$ at $37^{\circ} \mathrm{C}$, and cell debris was removed by centrifugation for $15 \mathrm{~min}$ at $1900 \times \mathrm{g}$. The clarified lysate was stored at $4{ }^{\circ} \mathrm{C}$ until the start of the purification, and vector titers were determined in the crude cell lysate using quantitative polymerase chain reaction (Q-PCR) with a primer directed against the promoter region of the specific transgene. Total to full ratio's for the productions were calculated by an optical density measurement using 260 and $280 \mathrm{~nm}$ as previously described [25].

\section{Vector purification}

The clarified lysate was filtered using a 0.22 um filter (Millipak 60, 0.22 um) and purified using AVB sepharose (GE Healthcare, Chicago, Illinois USA) in a fast protein liquid chromatography (FPLC) system (AKTA Explorer, GE Healthcare, Chicago, IL, USA). Purified virus titers were determined by Q-PCR.

\section{Densitometric analysis of the VP1:2:3 stoichiometry}

The protein composition of purified rAAV5 variants was determined by electrophoresis on $4-12 \%$ bis-tris polyacrylamide gels (Nupage, Life technologies, Carlsbad, CA, USA). Proteins were fixed for 30 min with $10 \% \mathrm{NaAC} / 7 \%$ $\mathrm{EtOH}$ and stained with Sypro Ruby (Life technologies) for $2 \mathrm{~h}$. Densitometric analysis of VP proteins was performed using Imagequant TL software (GE Healthcare) and the rolling ball algorithm. 


\section{In vitro potency}

Two continuous cell lines, Hela (ATCC) and Huh7 (JCRB), were infected with rAAV5-Seap variants at various MOI. WT Adenovirus was used at an MOI of 30 to stimulate second strand synthesis. Forty-eight hours after the start of the infection, Seap expression was measured in the supernatant using the Seap reporter assay kit (Roche, Bazel, Switzerland). Luminescence was measured on a Spectramax L luminometer (Molecular Devices, Sunnyvale, CA, USA) at $470 \mathrm{~nm}$ with an integration time of $1 \mathrm{~s}$. Error bars in the graphs represent the standard deviations and are based on three replicate seap activity measurements in the same experiment.

\section{In vivo potency, rodents}

Based on the in vitro results, the in vivo potency of Construct 11 was compared with that of Construct 2 and 13 (a previously reported chimeric AAV2/5 vector [7]) in mice. Briefly, C57BL/6 mice (Janvier labs, Le Genest-Saint-Isle, France) (male, juveline, 6-8 week old) were injected intravenously with Constructs 2, 11, and 13 containing hFIX as a reporter gene. In total, five groups of five mice each were injected with doses of $2 \times 10^{12}$ and $2 \times 10^{13} \mathrm{gc} /$ $\mathrm{kg}$; the control group was injected with vehicle (formulation buffer). A group size of five mice were selected for the in vivo study as historically we have consistently obtained statistically significant data from such a sample size. Statistical analysis was performed using one-way ANOVA. Citrated plasma was collected 1, 2, and 4 weeks following injection, after which the mice were sacrificed. Plasma levels of hFIX protein were measured using an hFIXspecific enzyme-linked immunosorbent assay (ELISA; VisuLize FIX antigen kit, Kordia, Leiden, the Netherlands). Optical density was measured at $450 \mathrm{~nm}$ on a Versamax ELISA plate reader (Molecular Devices, Sunnyvale, CA, USA). In vivo potency, NHP. This study was performed at Charles River Laboratories Preclinical Services (CRL), Edinburgh, UK. The animals used in the study were 3-4-year old, male cynomolgus macaques (Macaca fascicularis) weighing less than $6 \mathrm{~kg}$. In brief, three groups of three macaques received a single dose of Construct $2\left(5 \times 10^{12} \mathrm{gc} / \mathrm{kg}\right)$ or $11\left(5 \times 10^{11}\right.$ and $5 \times 10^{12} \mathrm{gc} /$ $\mathrm{kg}$ ) via slow-controlled $(30 \mathrm{~min})$ infusion into the saphenous vein. A group size of three macaques were selected to obtain meaningfull data while balancing ethical considerations. Plasma samples for analysis of circulating hFIX levels were taken 17 and 11 days before and $(8,14$, $21,28,56,84$, and 182 days) after dosing. Liver samples were taken at necropsy for evaluation of DNA delivery. All samples were analyzed by uniQure. Error bars in Fig. 5 represent standard error of mean.

\section{Vector DNA levels}

Total genomic DNA was extracted from frozen tissue powder and $250 \mathrm{ng}$ triplicates of each sample were subjected to Q-PCR using a primer-probe set specific for the rAAV5-hFIX vector genome present in Constructs 2 and 11. The number of vector DNA copies in each sample was calculated using a calibrator curve established by means of linear regression and serial dilutions of a plasmid containing the Q-PCR target sequence. The absence of inhibitors was verified by subjecting $250 \mathrm{ng}$ duplicates of each genomic DNA sample to Q-PCR using a primer-probe set specific for a sequence unique to the monkey porphobilinogen deaminase locus. Statistics was performed using a paired $t$ test.

\section{Circulating hFIX levels}

Circulating hFIX levels were assessed by sandwich ELISA. Plasmas were diluted 1/100 in assay buffer, then loaded onto ELISA wells coated with an hFIX-specific capture antibody (Kordia, Leiden, the Netherlands). Bound hFIX was detected using a horseradish peroxidaseconjugated FIX-specific detection antibody, followed by colorimetric substrate reaction and spectrophotometric readout. Levels of hFIX were quantified by means of interpolation from a calibrator curve, constructed from serial dilutions of a human calibrator plasma and nonlinear (four parameter) regression. Factor IX levels were averaged where errors bars in Fig. 5 represent a standard error of the mean.

\section{Infectivity assay}

The number of genome copies required for a single infectious particle was determined using a limiting dilutionbased infectious titer assay [12]. Briefly, HeLaRC32 (ATCC) cells stably expressing AAV-derived Rep and Cap were transduced with a series of rAAV5 variant dilutions in replicates of 10, and infected with or without WT adenovirus 5 (wtAd5) at a wtAd5:HeLaRC32 MOI of 50. Plates were incubated for $48 \mathrm{~h}$ at $37^{\circ} \mathrm{C}$ and wells were assessed for the presence or absence of replicated vector genome DNA by means of Q-PCR using a vector genome-specific primerprobe set. The number of infectious particles per seeded vector genome was calculated according to the Spearman-Karber method, using the fraction of positive wells for each dilution.

Animal studies were nonrandomized and nonblinded as outcomes were based on biochemical and molecular quantification data. All live animal experiments were vetted for compliance by an ethical committee of the respective CRO's. 
Acknowledgments The authors would like to thank Professor Sander van Deventer for his critical reading of the manuscript.

\section{Compliance with ethical standards}

Conflict of interest Financial support for this study was provided by uniQure. All authors of the manuscript are uniQure employees, and with the exception of Drs. Petry and de Haan, all receive stocks/ options. Dr. Lubelski is an author for other uniQure patent applications.

Open Access This article is licensed under a Creative Commons Attribution 4.0 International License, which permits use, sharing, adaptation, distribution and reproduction in any medium or format, as long as you give appropriate credit to the original author(s) and the source, provide a link to the Creative Commons license, and indicate if changes were made. The images or other third party material in this article are included in the article's Creative Commons license, unless indicated otherwise in a credit line to the material. If material is not included in the article's Creative Commons license and your intended use is not permitted by statutory regulation or exceeds the permitted use, you will need to obtain permission directly from the copyright holder. To view a copy of this license, visit http://creativecommons. org/licenses/by/4.0/.

\section{References}

1. van Oers MM. Opportunities and challenges for the baculovirus expression system. J Invertebr Pathol. 2011;107(Suppl):S3-15.

2. Urabe M, Ding C, Kotin RM. Insect cells as a factory to produce adeno-associated virus type 2 vectors. Hum Gene Ther. 2002;13:1935-43.

3. Trempe JP, Carter BJ. Alternate mRNA splicing is required for synthesis of adeno-associated virus VP1 capsid protein. J Virol. 1988;62:3356-63.

4. Cassinotti P, Weitz M, Tratschin JD. Organization of the adenoassociated virus (AAV) capsid gene: mapping of a minor spliced mRNA coding for virus capsid protein 1. Virology. 1988;167:176-84.

5. Smith RH, Levy JR, Kotin RM. A simplified baculovirus-AAV expression vector system coupled with one-step affinity purification yields high-titer rAAV stocks from insect cells. Mol Ther. 2009;17:1888-96.

6. Hermans W, Urabe M, Haast S AAV1 VP1changes. In: WO2007046703A2; 2007.

7. Urabe M, Nakakura T, Xin KQ, Obara Y, Mizukami H, Kume A, et al. Scalable generation of high-titer recombinant adenoassociated virus type 5 in insect cells. J Virol. 2006;80:1874-85.

8. Mietzsch M, Grasse S, Zurawski C, Weger S, Bennett A, Agbandje-McKenna M, et al. OneBac: platform for scalable and high-titer production of adeno-associated virus serotype 1-12 vectors for gene therapy. Hum Gene Ther. 2014;25:212-22.

9. Sonntag F, Bleker S, Leuchs B, Fischer R, Kleinschmidt JA. Adeno-associated virus type 2 capsids with externalized VP1/VP2 trafficking domains are generated prior to passage through the cytoplasm and are maintained until uncoating occurs in the nucleus. J Virol. 2006;80:11040-54.
10. Bleker S, Sonntag F, Kleinschmidt JA. Mutational analysis of narrow pores at the fivefold symmetry axes of adeno-associated virus type 2 capsids reveals a dual role in genome packaging and activation of phospholipase A2 activity. J Virol. 2005;79:2528-40.

11. Grieger JC, Johnson JS, Gurda-Whitaker B, Agbandje-McKenna M, Samulski RJ. Surface-exposed adeno-associated virus Vp1NLS capsid fusion protein rescues infectivity of noninfectious wild-type $\mathrm{Vp} 2 / \mathrm{Vp} 3$ and $\mathrm{Vp} 3$-only capsids but not that of fivefold pore mutant virions. J Virol. 2007;81:7833-43.

12. Grieger JC, Snowdy S, Samulski RJ. Separate basic region motifs within the adeno-associated virus capsid proteins are essential for infectivity and assembly. J Virol. 2006;80:5199-210.

13. Popa-Wagner R, Porwal M, Kann M, Reuss M, Weimer M, Florin $\mathrm{L}$, et al. Impact of VP1-specific protein sequence motifs on adenoassociated virus type 2 intracellular trafficking and nuclear entry. $\mathbf{J}$ Virol. 2012;86:9163-74.

14. Xiao PJ, Samulski RJ. Cytoplasmic trafficking, endosomal escape, and perinuclear accumulation of adeno-associated virus type 2 particles are facilitated by microtubule network. J Virol. 2012;86:10462-73.

15. Gao K, Li M, Zhong L, Su Q, Li J, Li S, et al. Empty virions in AAV8 vector preparations reduce transduction efficiency and may cause total viral particle dose-limiting side-effects. Mol Ther Methods Clin Dev. 2014;1:20139.

16. Girod A, Wobus CE, Zadori Z, Ried M, Leike K, Tijssen P, et al. The VP1 capsid protein of adeno-associated virus type 2 is carrying a phospholipase A2 domain required for virus infectivity. $\mathrm{J}$ Gen Virol. 2002;83(Pt 5):973-8.

17. Stahnke S, Lux K, Uhrig S, Kreppel F, Hosel M, Coutelle O, et al. Intrinsic phospholipase A2 activity of adeno-associated virus is involved in endosomal escape of incoming particles. Virology. 2011;409:77-83.

18. Kondratov O, Marsic D, Crosson SM, Mendez-Gomez HR, Moskalenko O, Mietzsch M, et al. Direct head-to-head evaluation of recombinant adeno-associated viral vectors manufactured in human versus insect cells. Mol Ther. 2017;25:2661-75.

19. Mietzsch M, Casteleyn V, Weger S, Zolotukhin S, Heilbronn R. OneBac 2.0: Sf9 cell lines for production of AAV5 vectors with enhanced infectivity and minimal encapsidation of foreign DNA. Hum Gene Ther. 2015;26:688-97.

20. Galibert L, Merten OW, van Oers MM, Riviere C. Improved baculovirus expression systems. In: WO/2013/014294; 2011.

21. Venkatakrishnan B, Yarbrough J, Domsic J, Bennett A, Bothner B, Kozyreva OG, et al. Structure and dynamics of adenoassociated virus serotype $1 \mathrm{VP1}$-unique $\mathrm{N}$-terminal domain and its role in capsid trafficking. J Virol. 2013;87:4974-84.

22. Hermans W, Urabe M, Haast S. Improved AAV vectors produced in insect cells. In: WO2007046703A2; 2007.

23. Ferreira JP, Noderer WL, Diaz de Arce AJ, Wang CL. Engineering ribosomal leaky scanning and upstream open reading frames for precise control of protein translation. Bioengineered. 2014;5:186-92.

24. Hinnebusch AG. Molecular mechanism of scanning and start codon selection in eukaryotes. Microbiol Mol Biol Rev. 2011;75:434-67

25. Sommer JM, Smith PH, Parthasarathy S, Isaacs J, Vijay S, Kieran $\mathrm{J}$, et al. Quantification of adeno-associated virus particles and empty capsids by optical density measurement. Mol Ther. 2003;7:122-8. 